\title{
Decálogo básico de la atención de la enfermedad de Chagas a nivel primario
}

\author{
Basic decalogue for care of Chagas's disease at primary level

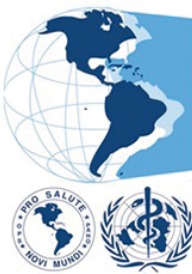 \\ Organización \\ Panamericana \\ de la Salud \\ Oficina Regional de la \\ Organización Mundial de la Salud
}

1. Las manifestaciones clínicas en la enfermedad de Chagas sólo permiten un diagnóstico presuntivo, y son las técnicas de laboratorio quiénes establecen un diagnóstico confirmatorio o de certeza.

2. Se debe tener en cuenta que el diagnóstico de laboratorio proactivo, es la principal vía de diagnóstico de las personas infectadas ya que la enfermedad de Chagas, en la mayor parte o durante toda su evolución, pasa desapercibida por ser asintomática u oligosintomática. Esto no implica que el paciente carezca de lesiones ya establecidas.

3. Se estima que 8 millones de personas en las Américas están infectadas por Trypanosoma cruzi y 28.000 se infectan por este microorganismo cada año. La mayoría queda sin diagnóstico, por falta de conocimiento clínico o por falta de medios, quedando consecuentemente sin tratamiento. Existe consenso que la mayoría de estos pacientes debe ser atendido en el primer nivel de atención, que cuente con recurso humano capacitado y tecnología adecuada y básica (ej. electrocardiógrafo), y un sistema de referencia y contra-referencia disponible.

4. Un cuadro de síndrome febril prolongado, cuando existe un antecedente clínico-epidemiológico, puede ser enfermedad de Chagas aguda o de reactivación, y deberán tomarse las medidas diagnósticas para confirmar o descartar esta hipótesis. Para el caso agudo de origen vectorial, oral o transfusional, deberán generar una respuesta sistémica que aborde prevención y control en la localidad de ocurrencia.

5. El diagnóstico de sospecha de enfermedad de Chagas debe plantearse por riesgo epidemiológico y/o cuadro clínico compatible. El riesgo epidemiológico debe contemplar la trasmisión vectorial (contacto con triatominos), la trasmisión por sangre y tejidos (transfusional, trasplante, congénita y uso de drogas inyectables intravenosas), transmisión oral (alimentos contaminados), accidente laboral (laboratorio, prácticas quirúrgicas), y existencia de familiares infectados (madre, hermanos).

6. Todo paciente con diagnóstico de Chagas, en cualquier etapa de su dolencia, debe recibir una completa atención acorde con las recomendaciones vigentes, que incluye el beneficio de recibir un tratamiento etiológico (tripanocida), correctamente indicado, controlado y evaluado. Asimismo, casos agudos, crónicos recientes, pediátricos, jóvenes y casos crónicos con reactivación por inmunocompromiso son indicación absoluta de tratamiento.

7. Los individuos infectados por Trypanosoma cruzi que recibieron un tratamiento etiológico correcto, pueden curar o mejorar sensiblemente su evolución, previniendo la morbi-mortalidad. El efecto curativo en niños y jóvenes, eliminándolos como reservorios, genera nuevas cohortes de futuros donantes de sangre $u$ órganos, y de gestantes libres de infección, previniendo la transmisión por estas vías.

8. La comunicación al paciente del diagnóstico y sus intervenciones terapéuticas, con sus atributos y limitaciones, debe ser clara, completa y prospectiva, dejando a la persona ubicada, consciente de su situación y necesidades de consulta y control. Es deseable la intervención de profesionales de apoyo (asistente social, psicólogo, etc.) para optimizar la calidad de la atención. 
9. El Chagas congénito puede controlarse, haciendo el diagnóstico y tratamiento oportuno en el recién nacido, hijo de madre infectada. Esto depende de incluir el tamizaje serológico universal en los controles del embarazo y el oportuno diagnóstico en el neonato.

10. Todo donante de sangre u órganos o personas captadas en estudios poblacionales, con tamizaje serológico positivo, debe ser informado y asesorado de su probable diagnóstico, y obligatoriamente derivado a consulta y/o servicio de referencia para confirmación/exclusión serológica, y en caso de confirmación diagnóstica definitiva, ejecución de completa atención médica.

La adecuada atención de las personas infectadas con Trypanosoma cruzi, se considera una acción estratégica esencial para el control integral de la Enfermedad de Chagas, dirigida hacia su objetivo final de eliminación como problema de Salud Pública.

Colonia del Sacramento, Uruguay 24 de abril de 2014

\section{Daniel Bulla}

Profesor Agregado de Clínica Médica. Encargado de Policlínica de Chagas. Facultad de Medicina. Hospital Maciel. Montevideo. Uruguay.

\section{Alejandro Luquetti}

Director del Laboratorio de Diagnóstico e Investigación de Enfermedad de Chagas. Universidad Federal de Goiás. Goiania. Brasil.

Martín Sánchez

Encargado de Policlínica de Chagas. Hospital de Artigas. MSP. Artigas. Uruguay.

Sergio Sosa Estani

Director. Instituto Nacional de Parasitología “Dr. Mario Fatala Chabén”. Buenos Aires. Argentina.

Roberto Salvatella Asesor Regional de Chagas OPS/OMS. Secretaría Técnica OPS/OMS. 